\title{
On para-Sasakian manifolds with a canonical paracontact connection
}

\author{
Bilal Eftal Acet and Selcen Yüksel Perktaş \\ Department of Mathematics, Faculty of Art and Science, Adiyaman University, Adiyaman, Turkey
}

Received: 28 January 2016, Accepted: 10 March 2016

Published online: 22 June 2016.

\begin{abstract}
The object of the present paper is to study a para-Sasakian manifold with a canonical paracontact connection. We prove that $\varphi$-conformally flat, $\varphi$-concircularly flat and $\varphi$-projectively flat para-Sasakian manifolds with respect to canonical paracontact connection are all $\eta$-Einstein manifolds. Also, it is shown that a quasi-concircularly flat para-Sasakian manifold is of constant scalar curvature.
\end{abstract}

Keywords: Para-Sasakian manifold, Canonical paracontact connetion.

\section{Introduction}

In 1976, I. Sato [7] introduced the notion of the almost paracontact structure $(\varphi, \xi, \eta)$ satisfying $\varphi^{2}=I-\eta \otimes \xi$ and $\eta(\xi)=1$ on a differentiable manifold and studied several properties of these manifolds (see also, [8]). The structure is an analogue of the almost contact structure ([2], [6]), and is closely related to almost product structure (in contrast to almost contact structure, which is related to almost complex structure). Every differentiable manifold with almost paracontact structure defined by I. Sato has a positive definite Riemannian metric.

S. Kaneyuki and M. Konzai [4] defined an almost paracontact structure on a pseudo-Riemannian manifold $M^{2 n+1}$ of dimension $(2 n+1)$ and constructed the almost paracomplex structure on $M^{2 n+1} \times \mathbb{R}$. Recently, S. Zamkovoy [18] has associated the almost paracontact structure given in [4] to a pseudo-Riemannian metric of signature $(n+1, n)$ and showed that any almost paracontact structure admits such a pseudo-Riemannian metric which is called compatible metric.

As a generalization of the well-known connection defined by N. Tanaka [10] and, independently, by S.M. Webster [12], in context of CR-geometry, Tanaka-Webster connection was introduced by S. Tanno [11]. In a paracontact metric manifold S. Zamkovoy [18] defined a canonical connection which plays the same role of the (generalized) Tanaka-Webster connection [11] in paracontact geometry (see also [3]). In this study we consider a canonical paracontact connection on a para-Sasakian manifold which seems to be the paracontact analogue of the (generalized) Tanaka-Webster connection.

In the present paper we study para-Sasakian manifolds with canonical paracontact connection. Section 2 is a general survey of the para-Sasakian manifolds. In section 3, we give some relations between curvature tensor (resp. Ricci tensor) with respect to canonical paracontact connection and curvature tensor (resp. Ricci tensor) with respect to Levi-Civita connection. In section $4, \varphi$-conformally flat para-Sasakian manifolds with respect to canonical paracontact connection 
are investigated. We study $\varphi$-concircularly flat para-Sasakian manifolds in Section 5 and show that a quasi-concircularly flat para-Sasakian manifold with respect to canonical paracontact connection is of constant scalar curvature. In Section 6, we obtain some characterizations for $\varphi-W_{2}$ flat para-Sasakian manifolds.

\section{Preliminaries}

Let $M^{2 n+1}$ be a differentiable manifold. If there exists a triple $(\varphi, \xi, \eta)$ of a tensor field $\varphi$ of type $(1,1)$, a vector field $\xi$ and a 1-form $\eta$ on $M^{2 n+1}$ which satisfy the relations [4]

$$
\begin{gathered}
\varphi^{2}=I-\eta \otimes \xi \\
\eta(\xi)=1, \quad \varphi \xi=0,
\end{gathered}
$$

where $I$ denotes the identity transformation, then we say the triple $(\varphi, \xi, \eta)$ is an almost paracontact structure and the manifold is an almost paracontact manifold. In this case the relations

$$
\eta \circ \varphi=0, \quad \operatorname{rank}(\varphi)=2 n
$$

hold. Moreover the tensor field $\varphi$ induces an almost paracomplex structure on the paracontact distribution $D=\operatorname{ker} \eta$, i.e. the eigendistributions $D^{ \pm}$corresponding to the eigenvalues \pm 1 of $\varphi$ are both $n$-dimensional.

If an almost paracontact manifold $M^{2 n+1}$ with an almost paracontact structure $(\varphi, \xi, \eta)$ admits a pseudo-Riemannian metric $g$ such that [18]

$$
g(\varphi X, \varphi Y)=-g(X, Y)+\eta(X) \eta(Y), \quad X, Y \in T M
$$

then we say that $M^{2 n+1}$ is an almost paracontact metric manifold with an almost paracontact metric structure $(\varphi, \xi, \eta, g)$ and such metric $g$ is called compatible metric. Any compatible metric $g$ is necessarily of signature $(n+1, n)$.

From (3) it is obvious that [18]

$$
\begin{gathered}
g(X, \varphi Y)=-g(\varphi X, Y), \\
g(X, \xi)=\eta(X),
\end{gathered}
$$

for any $X, Y \in T M^{2 n+1}$. The fundamental 2-form of $M^{2 n+1}$ is defined by

$$
\Phi(X, Y)=g(X, \varphi Y)
$$

An almost paracontact metric structure becomes a paracontact metric structure [18] if $g(X, \varphi Y)=d \eta(X, Y)$, for all vector fields $X, Y$, where $d \eta(X, Y)=\frac{1}{2}\{X \eta(Y)-Y \eta(X)-\eta([X, Y])\}$.

For an almost paracontact metric manifold $M^{2 n+1}$ with the structure $(\varphi, \xi, \eta, g)$, a local orthonormal basis can also be constructed as follows [18].

Let $U$ be coordinate neighborhood on $M^{2 n+1}$ and $X_{1}$ any unit vector field on $U$ orthogonal to $\xi$. Then $\varphi X_{1}$ is a vector field orthogonal to both $X_{1}$ and $\xi$, and $\left|\varphi X_{1}\right|^{2}=-1$. Now choose a unit vector field $X_{2}$ orthogonal to $\xi, X_{1}$ and $\varphi X_{1}$. Then $\varphi X_{2}$ is also vector field orthogonal to $\xi, X_{1}, \varphi X_{1}$ and $X_{2}$ and $\left|\varphi X_{2}\right|^{2}=-1$. Proceeding in this way we obtain a local 
orthonormal basis $\left(X_{i}, \varphi X_{i}, \xi\right),(i=1,2, \ldots, n)$ called a $\varphi$-basis.

We note that an almost paracomplex structure on a $2 n$-dimensional manifold, denote by $J$, is a $(1,1)$-type tensor satisfying $J^{2}=I$ and it has eigensubbundles $T^{+}$and $T^{-}$corresponding to the eigenvalues $1,-1$ of $J$ respectively. The Nijenhuis tensor $N$ of $J$, given by

$$
N_{J}(X, Y)=[J X, J Y]+[X, Y]-J[J X, Y]-J[X, J Y],
$$

is the obstruction for the integrability of the eigensubbundles $T^{+}, T^{-}$. If $N=0$, then the almost paracomplex structure is called paracomplex or integrable [16].

Let $M^{2 n+1}$ be an almost paracontact metric manifold with structure $(\varphi, \xi, \eta)$ and consider the manifold $M^{2 n+1} \times \mathbb{R}$. We denote a vector field on $M^{2 n+1} \times \mathbb{R}$, by $\left(X, f \frac{d}{d t}\right)$ where $X$ is tangent to $M^{2 n+1}, t$ is the coordinate on $\mathbb{R}$ and $f$ is a $C^{\infty}$ function on $M^{2 n+1} \times \mathbb{R}$. An almost paracomplex structure $J$ on $M^{2 n+1} \times \mathbb{R}$ is defined by [5]

$$
J\left(X, f \frac{d}{d t}\right)=\left(\varphi X+f \xi, \eta(X) \frac{d}{d t}\right) .
$$

If $J$ is integrable we say that the almost paracontact structure $(\varphi, \xi, \eta)$ is normal. A normal paracontact metric manifold is a para-Sasakian manifold. An almost paracontact metric structure $(\varphi, \xi, \eta, g)$ on a $M^{2 n+1}$ is para-Sasakian manifold if and only if [18]

$$
\left(\nabla_{X} \varphi\right) Y=-g(X, Y) \xi+\eta(Y) X,
$$

where $X, Y \in T M^{2 n+1}$ and $\nabla$ is Levi-Civita connection of $M^{2 n+1}$.

From (6), it can be seen that

$$
\nabla_{X} \xi=-\varphi X
$$

Example 1. [1]Let $M=\mathbb{R}^{2 n+1}$ be the $(2 n+1)$-dimensional real number space with $\left(x_{1}, y_{1}, x_{2}, y_{2}, \ldots, x_{n}, y_{n}, z\right)$ standard coordinate system. Defining

$$
\begin{aligned}
\varphi \frac{\partial}{\partial x_{\alpha}} & =\frac{\partial}{\partial y_{\alpha}}, \quad \varphi \frac{\partial}{\partial y_{\alpha}}=\frac{\partial}{\partial x_{\alpha}}, \quad \varphi \frac{\partial}{\partial z}=0 \\
\xi & =\frac{\partial}{\partial z}, \quad \eta=d z \\
g & =\eta \otimes \eta+\sum_{\alpha=1}^{n} d x_{\alpha} \otimes d x_{\alpha}-\sum_{\alpha=1}^{n} d y_{\alpha} \otimes d y_{\alpha}
\end{aligned}
$$

where $\alpha=1,2, \ldots, n$, then the set $(M, \varphi, \xi, \eta, g)$ is an almost paracontact metric manifold.

Furthermore, in a para-Sasakian manifold $M^{2 n+1}$, the following relations hold [18]:

$$
\begin{gathered}
g(R(X, Y) Z, \xi)=\eta(R(X, Y) Z)=g(X, Z) \eta(Y)-g(Y, Z) \eta(X), \\
R(X, Y) \xi=\eta(X) Y-\eta(Y) X \\
R(\xi, X) Y=-g(X, Y) \xi+\eta(Y) X
\end{gathered}
$$




$$
\begin{gathered}
R(\xi, X) \xi=X-\eta(X) \xi \\
S(X, \xi)=-2 n \eta(X),
\end{gathered}
$$

for any vector fields $X, Y, Z \in T M^{2 n+1}$. Here, $R$ is Riemannian curvature tensor and $S$ is Ricci tensor defined by $S(X, Y)=g(Q X, Y)$ where $Q$ is Ricci operator.

Now we consider the connection $\bar{\nabla}$ defined by [11],

$$
\bar{\nabla}_{X} Y=\nabla_{X} Y+\eta(X) \varphi Y-\eta(Y) \nabla_{X} \xi+\left(\nabla_{X} \eta\right) Y \cdot \xi
$$

where $X, Y \in T M^{2 n+1}$ and $\nabla$ denotes Levi-Civita connection on $M^{2 n+1}$. If we use equation (7) in (13), then we obtain

$$
\bar{\nabla}_{X} Y=\nabla_{X} Y+\eta(X) \varphi Y+\eta(Y) \varphi X+g(X, \varphi Y) \xi
$$

Definition 1. We call the connection $\bar{\nabla}$ defined by (14) on a para-Sasakian manifold the canonical paracontact connection on a para-Sasakian manifold.

Proposition 1. On a para-Sasakian manifold the connection $\bar{\nabla}$ has the following properties:

$$
\begin{gathered}
\bar{\nabla} \eta=0, \quad \bar{\nabla} g=0, \quad \bar{\nabla} \xi=0, \\
\left(\bar{\nabla}_{X} \varphi\right) Y=\left(\nabla_{X} \varphi\right) Y+g(X, Y) \xi-\eta(Y) X .
\end{gathered}
$$

Proof. Calculation is straightforward by using (14).

\section{Curvature tensor}

The curvature tensor $\bar{R}$ of a para-Sasakian manifold $M$ with respect to the canonical paracontact connection $\bar{\nabla}$ is defined by

$$
\bar{R}(X, Y) Z=\bar{\nabla}_{X} \bar{\nabla}_{Y} Z-\bar{\nabla}_{Y} \bar{\nabla}_{X} Z-\bar{\nabla}_{[X, Y]} Z
$$

By using equation (14) in (17), we obtain

$$
\begin{aligned}
\bar{R}(X, Y) Z & =R(X, Y) Z+g(Y, Z) \eta(X) \xi-g(X, Z) \eta(Y) \xi \\
& +\eta(Y) \eta(Z) X-\eta(X) \eta(Z) Y+2 g(X, \varphi Y) \varphi Z \\
& +g(X, \varphi Z) \varphi Y-g(Y, \varphi Z) \varphi X
\end{aligned}
$$

where $R(X, Y) Z=\nabla_{X} \nabla_{Y} Z-\nabla_{Y} \nabla_{X} Z-\nabla_{[X, Y]} Z$ is the curvature tensor of $M$ with respect to Levi-Civita connection $\nabla$.

Let $T$ and $\bar{T}$ be curvature tensors of type $(0,4)$ given by

$$
T(X, Y, Z, W)=g(R(X, Y) Z, W)
$$

and

$$
\bar{T}(X, Y, Z, W)=g(\bar{R}(X, Y) Z, W)
$$


respectively.

Theorem 1. In a para-Sasakian manifold the following relations hold:

$$
\begin{gathered}
\bar{R}(X, Y) Z+\bar{R}(Y, Z) X+\bar{R}(Z, X) Y=0, \\
\bar{T}(X, Y, Z, W)+\bar{T}(Y, X, Z, W)=0 \\
\bar{T}(X, Y, Z, W)+\bar{T}(X, Y, W, Z)=0, \\
\bar{T}(X, Y, Z, W)-\bar{T}(Z, W, X, Y)=0 .
\end{gathered}
$$

Proof. Using (18) and first Bianchi identity with respect to Levi-Civita connection $\nabla$, we obtain

$$
\begin{aligned}
\bar{R}(X, Y) Z+\bar{R}(Y, Z) X+\bar{R}(Z, X) Y & =2 g(X, \varphi Y) \varphi Z+g(X, \varphi Z) \varphi Y-g(Y, \varphi Z) \varphi X+2 g(Y, \varphi Z) \varphi X \\
& +g(Y, \varphi X) \varphi Z-g(Z, \varphi X) \varphi Y+2 g(Z, \varphi X) \varphi Y+g(Z, \varphi Y) \varphi X \\
& -g(X, \varphi Y) \varphi Z .
\end{aligned}
$$

If we use (4) in (23) we get (19). Next, from (18) we have

$$
\begin{aligned}
\bar{T}(X, Y, Z, W) & =T(X, Y, Z, W)+g(Y, Z) \eta(X) \eta(W)-g(X, Z) \eta(Y) \eta(W)+g(X, W) \eta(Y) \eta(Z) \\
& -g(Y, W) \eta(X) \eta(Z)+2 g(X, \varphi Y) g(\varphi Z, W)+g(X, \varphi Z) g(\varphi Y, W)-g(Y, \varphi Z) g(\varphi X, W) .
\end{aligned}
$$

It is well known that

$$
T(X, Y, Z, W)=-T(Y, X, Z, W), T(X, Y, Z, W)=T(X, Y, W, Z), \quad T(X, Y, Z, W)=T(Z, W, X, Y) .
$$

By taking into account above equations we get (20), (21) and (22), respectively.

Let $E_{i}=\left\{e_{i}, \varphi e_{i}, \xi\right\}(i=1,2, \ldots, n)$ be a local orthonormal $\varphi$-basis of a para-Sasakian manifold $M$. Then the Ricci tensor $\bar{S}$ and the scalar curvature $\bar{\tau}$ of $M$ with respect to canonical paracontact connection $\bar{\nabla}$ are defined by

$$
\bar{S}(X, Y)=\sum_{i=1}^{n} g\left(\bar{R}\left(e_{i}, X\right) Y, e_{i}\right)-\sum_{i=1}^{n} g\left(\bar{R}\left(\varphi e_{i}, X\right) Y, \varphi e_{i}\right)+g(\bar{R}(\xi, X) Y, \xi),
$$

and

$$
\bar{\tau}=\sum_{j=1}^{n} \bar{S}\left(e_{j}, e_{j}\right)-\sum_{j=1}^{n} \bar{S}\left(\varphi e_{j}, \varphi e_{j}\right)+\bar{S}(\xi, \xi)
$$

respectively.

Theorem 2. In a para-Sasakian manifold $M$, the Ricci tensor $\bar{S}$ and scalar curvature $\bar{\tau}$ of canonical paracontact connection $\bar{\nabla}$ are defined by

$$
\begin{gathered}
\bar{S}(X, Y)=S(X, Y)-2 g(X, Y)+(2 n+2) \eta(X) \eta(Y), \\
\bar{\tau}=\tau-2 n,
\end{gathered}
$$

where $S$ and $\tau$ denote the Ricci tensor and scalar curvature of Levi-Civita connection $\nabla$, respectively. Consequently, $\bar{S}$ is symmetric. 
Corollary 1. If a para-Sasakian manifold is Ricci-flat with respect to canonical paracontact connection then it is an $\eta$-Einstein manifold.

Lemma 1. Let M be a para-Sasakian manifold with canonical paracontact connection $\bar{\nabla}$. Then

$$
\begin{gathered}
g(\bar{R}(X, Y) Z, \xi)=\eta(\bar{R}(X, Y) Z)=0 \\
\bar{R}(X, Y) \xi=\bar{R}(\xi, X) Y=\bar{R}(\xi, X) \xi=0 \\
\bar{S}(X, \xi)=0
\end{gathered}
$$

for all $X, Y, Z \in T M$.

\section{Conformal curvature tensor with canonical paracontact connection}

The conformal curvature tensor (see [17]) of a para-Sasakian manifold $M$ with respect to canonical paracontact connection is defined by

$$
\bar{C}(X, Y) V=\bar{R}(X, Y) V-\frac{1}{2 n-1}\left(\begin{array}{c}
\bar{S}(Y, V) X-\bar{S}(X, V) Y \\
+g(Y, V) \bar{Q} X-g(X, V) \bar{Q} Y
\end{array}\right)+\frac{\bar{\tau}}{2 n(2 n-1)}(g(Y, V) X-g(X, V) Y)
$$

for any vector fields $X, Y, V \in T M$.

Using (18), (27) and (28) in (32), we have

$$
\begin{aligned}
\bar{C}(X, Y) V & =R(X, Y) V+g(Y, V) \eta(X) \xi-g(X, V) \eta(Y) \xi+\eta(Y) \eta(V) X-\eta(X) \eta(V) Y+2 g(X, \varphi Y) \varphi V \\
& +g(X, \varphi V) \varphi Y-g(Y, \varphi V) \varphi X-\frac{1}{2 n-1} A+\frac{\tau-2 n}{2 n(2 n-1)}(g(Y, V) X-g(X, V) Y)
\end{aligned}
$$

where

$$
A=\left(\begin{array}{c}
S(Y, V) X-S(X, V) Y \\
-4 g(Y, V) X+4 g(X, V) Y \\
+g(Y, V) Q X-g(X, V) Q Y \\
+(2 n+2)\left[\begin{array}{c}
\eta(Y) \eta(V) X-\eta(X) \eta(V) Y \\
+g(Y, V) \eta(X) \xi-g(X, V) \eta(Y) \xi
\end{array}\right]
\end{array}\right)
$$

Definition 2. A differentiable manifold $M$ satisfying the condition

$$
\varphi^{2} \bar{C}(\varphi X, \varphi Y) \varphi V=0
$$

is called $\varphi$-conformally flat (see [13]).

It can be easily seen that $\varphi^{2} \bar{C}(\varphi X, \varphi Y) \varphi V=0$ holds if and only if

$$
g(\bar{C}(\varphi X, \varphi Y) \varphi V, \varphi U)=0
$$

for any $X, Y, U, V \in T M$. 
In view of (33), $\varphi$-conformally flatness implies that

$$
\begin{aligned}
& g(R(\varphi X, \varphi Y) \varphi V, \varphi U)+2 g(\varphi X, Y) g(V, \varphi U)+g(\varphi X, V) g(Y, \varphi U)-g(\varphi Y, V) g(X, \varphi U) \\
& =\frac{1}{2 n-1} B-\frac{\tau-2 n}{2 n(2 n-1)}\left(\begin{array}{c}
g(\varphi Y, \varphi V) g(\varphi X, \varphi U) \\
-g(\varphi X, \varphi V) g(\varphi Y, \varphi U)
\end{array}\right) .
\end{aligned}
$$

where

$$
B=\left(\begin{array}{c}
S(\varphi Y, \varphi V) g(\varphi X, \varphi U)-S(\varphi X, \varphi V) g(\varphi Y, \varphi U) \\
-2 g(\varphi Y, \varphi V) g(\varphi X, \varphi U)+2 g(\varphi X, \varphi V) g(\varphi Y, \varphi U) \\
+S(\varphi X, \varphi U) g(\varphi Y, \varphi V)-S(\varphi Y, \varphi U) g(\varphi X, \varphi V) \\
-2 g(\varphi Y, \varphi V) g(\varphi X, \varphi U)+2 g(\varphi X, \varphi V) g(\varphi Y, \varphi U)
\end{array}\right)
$$

Choosing $\left\{e_{i}, \varphi e_{i}, \xi\right\}$ as an orthonormal basis of vector fields in $M$, by a suitable contraction of (36) with respect to $X$ and $U$ we obtain

$$
S(\varphi Y, \varphi V)-2 g(\varphi Y, \varphi V)=\frac{1}{2 n-1}\left(\begin{array}{c}
(2 n-2) S(\varphi Y, \varphi V) \\
+(\tau+4-6 n) g(\varphi Y, \varphi V)
\end{array}\right)-\frac{\tau-2 n}{2 n(2 n-1)}((2 n-1) g(\varphi Y, \varphi V)),
$$

for any vector fields $Y, V \in T M$. From the last equation above, we get

$$
S(Y, V)=-\left(\frac{\tau+2 n}{2 n}\right) g(Y, V)+\left(\frac{\tau-4 n^{2}+2 n}{2 n}\right) \eta(Y) \eta(V),
$$

which implies that $M$ is an $\eta$-Einstein manifold. Therefore we have the following.

Theorem 3. Let $M$ be a $\varphi$-conformally flat para-Sasakian manifold with respect to canonical paracontact connection. Then $M$ is an $\eta-$ Einstein manifold.

Definition 3. A differentiable manifold $M$ satisfying the condition

$$
g(\bar{C}(X, Y) V, \varphi U)=0,
$$

is called quasi-conformally flat.

From (33) we can write

$$
\begin{aligned}
& g(R(X, Y) V, \varphi U)+g(X, \varphi U) \eta(Y) \eta(V)-g(Y, \varphi U) \eta(X) \eta(V)+2 g(X, \varphi Y) g(\varphi V, \varphi U) \\
& +g(X, \varphi V) g(\varphi Y, \varphi U)-g(Y, \varphi V) g(\varphi X, \varphi U)=\frac{1}{2 n-1} C-\frac{\tau-2 n}{2 n(2 n-1)}\left(\begin{array}{c}
g(Y, V) g(X, \varphi U) \\
-g(X, V) g(Y, \varphi U)
\end{array}\right) .
\end{aligned}
$$

where

$$
C=\left(\begin{array}{c}
S(Y, V) g(X, \varphi U)-S(X, V) g(Y, \varphi U) \\
-4 g(Y, V) g(X, \varphi U)+4 g(X, V) g(Y, \varphi U) \\
+S(X, \varphi U) g(Y, V)-S(Y, \varphi U) g(X, V) \\
+(2 n+2)\left[\begin{array}{c}
g(X, \varphi U) \eta(Y) \eta(V) \\
-g(Y, \varphi U) \eta(X) \eta(V)
\end{array}\right]
\end{array}\right) .
$$

Putting $Y=V=\xi$ in (39) and by using (1) we get

$$
g(R(X, \xi) \xi, \varphi U)+g(X, \varphi U)=\frac{1}{2 n-1}(S(X, \varphi U)-2 g(X, \varphi U))-\frac{\tau-2 n}{2 n(2 n-1)} g(X, \varphi U),
$$


for any vector fields $X$ and $U$ on $M$.

In view of (11), we obtain

$$
S(X, \varphi U)=\left(\frac{\tau+2 n}{2 n}\right) g(X, \varphi U)
$$

Replacing $U$ by $\varphi U$ in the last equation we have

$$
S(X, U)=\left(\frac{\tau+2 n}{2 n}\right) g(X, U)-\left(\frac{\tau+2 n+4 n^{2}}{2 n}\right) \eta(X) \eta(U) .
$$

Therefore we have the following.

Theorem 4. Let $M$ be a quasi-conformally flat para-Sasakian manifold with respect to canonical paracontact connection. Then $M$ is an $\eta$-Einstein manifold.

\section{Concircular Curvature tensor with canonical paracontact connection}

The concircular curvature tensor (see [14],[15]) of a $(2 n+1)$-dimensional para-Sasakian manifold $M$ with respect to canonical paracontact connection is defined by;

$$
\bar{Z}(X, Y) V=\bar{R}(X, Y) V-\frac{\bar{\tau}}{2 n(2 n-1)}(g(Y, V) X-g(X, V) Y)
$$

By using (18) and (28), we obtain from (41)

$$
\begin{aligned}
\bar{Z}(X, Y) V & =R(X, Y) V+g(Y, V) \eta(X) \xi-g(X, V) \eta(Y) \xi+\eta(Y) \eta(V) X-\eta(X) \eta(V) Y+2 g(X, \varphi Y) \varphi V \\
& +g(X, \varphi V) \varphi Y-g(Y, \varphi V) \varphi X-\frac{\tau-2 n}{2 n(2 n-1)}(g(Y, V) X-g(X, V) Y) .
\end{aligned}
$$

Definition 4. A differentiable manifold $M$ satisfying the condition

$$
\varphi^{2} \bar{Z}(\varphi X, \varphi Y) \varphi V=0
$$

is called $\varphi$-concircularly flat.

It can be easily seen that $\varphi^{2} \bar{Z}(\varphi X, \varphi Y) \varphi V=0$ holds if and only if

$$
g(\bar{Z}(\varphi X, \varphi Y) \varphi V, \varphi U)=0
$$

for any $X, Y, U, V \in T M$.

Using (42), $\varphi$-concircularly flatness means

$$
\begin{aligned}
g(R(\varphi X, \varphi Y) \varphi V, \varphi U)+ & 2 g(\varphi X, Y) g(V, \varphi U)+g(\varphi X, V) g(Y, \varphi U)-g(\varphi Y, V) g(X, \varphi U) \\
= & \frac{\tau-2 n}{2 n(2 n-1)}\left(\begin{array}{c}
g(\varphi Y, \varphi V) g(\varphi X, \varphi U) \\
-g(\varphi X, \varphi V) g(\varphi Y, \varphi U)
\end{array}\right)
\end{aligned}
$$

Choosing $\left\{e_{i}, \varphi e_{i}, \xi\right\}$ as an orthonormal basis of vector fields in $M$, so by suitable contraction of (45) with respect to $X$ and $U$ we obtain

$$
S(\varphi Y, \varphi V)-2 g(\varphi Y, \varphi V)=-\frac{\tau-2 n}{2 n(2 n-1)}((2 n-1) g(\varphi Y, \varphi V))
$$


for any vector fields $Y$ and $V$ on $M$. From above equation, we get

$$
S(Y, V)=-\left(\frac{\tau+2 n}{2 n}\right) g(Y, V)+\left(\frac{\tau-4 n^{2}+2 n}{2 n}\right) \eta(Y) \eta(V),
$$

which implies that $M$ is an $\eta$-Einstein manifold. Therefore we have:

Theorem 5. Let $M$ be a $\varphi$-concircularly flat para-Sasakian manifold with respect to canonical paracontact connection. Then $M$ is an $\eta$-Einstein manifold.

Definition 5. A differentiable manifold $M$ satisfying the condition

$$
g(\bar{Z}(X, Y) V, \varphi U)=0,
$$

is called quasi-concircularly flat.

From (42), we can write

$$
\begin{aligned}
g(R(X, Y) V, \varphi U)+ & g(X, \varphi U) \eta(Y) \eta(V)-g(Y, \varphi U) \eta(X) \eta(V)+2 g(X, \varphi Y) g(\varphi V, \varphi U) \\
& +g(X, \varphi V) g(\varphi Y, \varphi U)-g(Y, \varphi V) g(\varphi X, \varphi U)=\frac{\tau-2 n}{2 n(2 n-1)}\left(\begin{array}{c}
g(Y, V) g(X, \varphi U) \\
-g(X, V) g(Y, \varphi U)
\end{array}\right)
\end{aligned}
$$

Putting $Y=V=\xi$ in (47) and by using (1) we get

$$
g(R(X, \xi) \xi, \varphi U)+g(X, \varphi U)=\frac{\tau-2 n}{2 n(2 n-1)} g(X, \varphi U),
$$

for any vector fields $X$ and $U$ on $M$. By use of (11) we obtain

$$
\left(\frac{\tau-2 n}{2 n(2 n-1)}\right) g(X, \varphi U)=0
$$

From above equation we can state following:

Theorem 6. If a para-Sasakian manifold $M$ is quasi-concircularly flat with respect to canonical paracontact connection, then it is of constant scalar curvature.

\section{Projective curvature tensor with canonical paracontact connection}

Let $M$ be a para-Sasakian manifold with canonical paracontact connection. The projective curvature tensor (see [17]) of $M$ is defined by;

$$
\bar{P}(X, Y) V=\bar{R}(X, Y) V-\frac{1}{2 n}(\bar{S}(Y, V) X-\bar{S}(X, V) Y) .
$$

By using (18) and (27), we obtain from (49)

$$
\begin{aligned}
& \bar{P}(X, Y) V=R(X, Y) V+g(Y, V) \eta(X) \xi-g(X, V) \eta(Y) \xi+\eta(Y) \eta(V) X-\eta(X) \eta(V) Y+2 g(X, \varphi Y) \varphi V \\
& +g(X, \varphi V) \varphi Y-g(Y, \varphi V) \varphi X-\frac{1}{2 n}\left(\begin{array}{c}
S(Y, V) X-S(X, V) Y \\
-2 g(Y, V) X+2 g(X, V) Y \\
+(2 n+2)[\eta(Y) \eta(V) X-\eta(X) \eta(V) Y]
\end{array}\right) .
\end{aligned}
$$


Definition 6. A differentiable manifold $M$ satisfying the condition

$$
\varphi^{2} \bar{P}(\varphi X, \varphi Y) \varphi V=0
$$

is called $\varphi$-projectively flat.

It can be easily seen that $\varphi^{2} \bar{P}(\varphi X, \varphi Y) \varphi V=0$ holds if and only if

$$
g(\bar{P}(\varphi X, \varphi Y) \varphi V, \varphi U)=0
$$

for any $X, Y, U, V \in T M$.

In view of (50), if $M$ is $\varphi$-projectively flat then we have

$$
\begin{aligned}
g(R(\varphi X, \varphi Y) \varphi V, \varphi U)+ & 2 g(\varphi X, Y) g(V, \varphi U)+g(\varphi X, V) g(Y, \varphi U)-g(\varphi Y, V) g(X, \varphi U) \\
= & \frac{1}{2 n}\left(\begin{array}{c}
S(\varphi Y, \varphi V) g(\varphi X, \varphi U)-S(\varphi X, \varphi V) g(\varphi Y, \varphi U) \\
-2 g(\varphi Y, \varphi V) g(\varphi X, \varphi U)+2 g(\varphi X, \varphi V) g(\varphi Y, \varphi U)
\end{array}\right)
\end{aligned}
$$

Choosing $\left\{e_{i}, \varphi e_{i}, \xi\right\}$ as an orthonormal basis of vector fields in $M$, so by suitable contraction of (53) with respect to $X$ and $U$ we obtain

$$
S(\varphi Y, \varphi V)-2 g(\varphi Y, \varphi V)=\frac{1}{2 n}\left(\begin{array}{c}
(2 n-1) S(\varphi Y, \varphi V) \\
+(2-4 n) g(\varphi Y, \varphi V)
\end{array}\right)
$$

for any vector fields $Y$ and $V$ on $M$. From above equation, we get

$$
S(Y, V)=-2 g(Y, V)+(2-2 n) \eta(Y) \eta(V),
$$

which implies that $M$ is an $\eta$-Einstein manifold. Hence we have the following.

Theorem 7. Let $M$ be a $\varphi$-projectively flat para-Sasakian manifold with respect to canonical paracontact connection. Then it is an $\eta$-Einstein manifold.

Definition 7. A differentiable manifold $M$ satisfying the condition

$$
g(\bar{P}(X, Y) V, \varphi U)=0
$$

is called quasi-projectively flat.

From (50) we can write

$$
\begin{aligned}
g(R(X, Y) V, \varphi U) & +g(X, \varphi U) \eta(Y) \eta(V)-g(Y, \varphi U) \eta(X) \eta(V)+2 g(X, \varphi Y) g(\varphi V, \varphi U) \\
& +g(X, \varphi V) g(\varphi Y, \varphi U)-g(Y, \varphi V) g(\varphi X, \varphi U)=\frac{1}{2 n} S R \eta
\end{aligned}
$$

where

$$
S R \eta=\frac{1}{2 n}\left(\begin{array}{c}
S(Y, V) g(X, \varphi U)-S(X, V) g(Y, \varphi U) \\
-2 g(Y, V) g(X, \varphi U)+2 g(X, V) g(Y, \varphi U) \\
+(2 n+2)\left[\begin{array}{c}
g(X, \varphi U) \eta(Y) \eta(V) \\
-g(Y, \varphi U) \eta(X) \eta(V)
\end{array}\right]
\end{array}\right)
$$


Let $\left\{e_{i}, \varphi e_{i}, \xi\right\}(i=1, \ldots, n)$ be an orthonormal basis of the tangent space at any point. So a contraction of (56) with respect to $Y$ and $V$ gives

$$
S(X, \varphi U)-g(R(X, \xi) \xi, \varphi U)+3 g(X, \varphi U)=\frac{1}{2 n}(-S(X, \varphi U)+(\tau-2 n+4) g(X, \varphi U)),
$$

for any vector fields $X$ and $U$ on $M$.

In view of (11) we obtain

$$
S(X, \varphi U)=\left(\frac{\tau-10 n+4}{2 n+1}\right) g(X, \varphi U) .
$$

Replacing $U$ by $\varphi U$ in the above equation we have

$$
S(X, U)=\left(\frac{\tau-10 n+4}{2 n+1}\right) g(X, U)-\left(\frac{\tau-8 n+4+4 n^{2}}{2 n+1}\right) \eta(X) \eta(U) .
$$

Therefore we have the following:

Theorem 8. Let $M$ be a quasi-projectively flat para-Sasakian manifold with respect to canonical paracontact connection. Then $M$ is an $\eta$-Einstein manifold.

\section{Conclusion}

In this manuscript we study canonical paracontact connection on para-Sasakian manifolds. Also we obtain some curvature conditions on para-Sasakian manifolds with a canonical paracontact connection.

\section{References}

[1] B. E. Acet, S. Yüksel Perktaş, E. Kılıç, On lightlike geometry of para-Sasakian manifolds, Scientific Work J., Article ID 696231 , 2014.

[2] D. E. Blair, Contact manifolds in Riemannian geometry, Lecture Notes in Mathematics, Vol. 509. Springer-Verlag, Berlin-New York, 1976.

[3] B. E. Acet, E. Kiliç, S. Yüksel Perktaş, Some Curvature Conditions on a Para-Sasakian Manifold with Canonical Paracontact Connection, Int. J. of Math. and Math. Sci., Article ID 395462, 2012.

[4] S. Kaneyuki, M. Konzai, Paracomplex structure and affine symmetric spaces, Tokyo J. Math., 8 (1985), 301-318.

[5] S. Kaneyuki, F. L. Willams, Almost paracontact and parahodge structure on manifolds, Nagoya Math. J., 99 (1985), $173-187$.

[6] S. Sasaki, On differentiable manifolds with certain structures which are closely related to almost contact structure I, Tôhoku Math. J., 12 (1960), 459-476.

[7] I. Sato, On a structure similar to the almost contact structure I., Tensor N. S., 30 (1976), 219-224.

[8] I. Sato, On a structure similar to the almost contact structure II., Tensor N. S., 31 (1977), 199-205.

[9] T. Takahashi, Sasakian manifold with pseudo-Riemannian metric, Tôhoku Math. J., 21 (1969), 644-653.

[10] N. Tanaka, On non-degenerate real hypersurfaces, graded Lie algebras and Cartan connection, Japan J. Math., 2 (1976), $131-190$.

[11] S. Tanno, Variational problems on contact Riemannian manifolds, Trans. Amer. Math. Soc., 314 (1989), 349-379.

[12] S. M. Webster, Pseudo-Hermitian structures on a real hypersurfaces, J. Diff. Geo., 13 (1979), 25-41.

[13] J. L. Cabrerizo, L. M. Fernandez, M. Fernandez, G. Zhen, The structuren of a class of K-contact manifolds, Acta Math. Hungarica, 82 (1999), 331-340.

[14] K. Yano, Concircular geometry I , Concircular transformations, Proc. Imp. Acad.Tokyo, 16 (1940), 195-200.

[15] K. Yano, S. Bochner, Curvature and Betti numbers, Annals of Mathematics Studies 32, Princeton University Press, 1953. 
[16] K. Yano, Affine connexions in almost product spaces, Kodai Math. Sem. Rep., 11 (1959), 1-24.

[17] K. Yano, M. Kon, Structures on Manifolds, Series in Pure Math., Vol 3,World Sci, 1984.

[18] S. Zamkovoy, Canonical connection on paracontact manifolds, Ann. Glob. Anal. Geo., 36 (2009), 37-60. 